\title{
The role of triiodothyronine (T3) and T3/free thyroxine (fT4) in glucose metabolism during pregnancy: the Ma'anshan birth cohort study
}

\author{
Beibei Zhu' 1,2,3,4, Yan Han ${ }^{1,2,3,4}$, Fen Deng ${ }^{1,2,3,4}$, Kun Huang1,2,3,4, Shuangqin Yan ${ }^{5}$, Jiahu Hao ${ }^{1,2,3,4}$, Peng Zhu ${ }^{1,2,3,4}$ and \\ Fangbiao Tao 1 1,2,3,4 \\ ${ }^{1}$ School of Public Health, Anhui Medical University, Hefei, Anhui, China \\ ${ }^{2}$ Key Laboratory of Population Health Across Life Cycle, Anhui Medical University, Ministry of Education of the People's Republic of China, Hefei, \\ Anhui, China \\ ${ }^{3} \mathrm{NHC}$ Key Laboratory of Study on Abnormal Gametes and Reproductive Tract, Hefei, Anhui, China \\ ${ }^{4}$ Anhui Provincial Key Laboratory of Population Health and Aristogenics, Anhui Medical University, Hefei, Anhui, China \\ ${ }^{5} \mathrm{Ma}$ 'anshan Maternal and Child Health Care Center, Ma'anshan, Anhui, China
}

Correspondence should be addressed to F Tao: taofangbiao@126.com

\begin{abstract}
Objectives: Compared with other thyroid markers, fewer studies have explored the associations between triiodothyronine (T3), T3/free thyroxine (fT4) and glucose abnormality during pregnancy. Thus, we aimed to: (i) examine the associations of T3 and T3/fT4 with glucose metabolism indicators and (ii) evaluate, in the first trimester, the performance of the two markers as predictors of gestational diabetes mellitus (GDM) risk.

Methods: Longitudinal data from 2723 individuals, consisting of three repeated measurements of T3 and fT4, from the Man'anshan birth cohort study (MABC), China, were analyzed using a time-specific generalized estimating equation (GEE). The receiver operating characteristic curve (ROC) - area under the curve (AUC) and HosmerLemeshow goodness of fit test was used to assess the discrimination and calibration of prediction models.

Results: T3 and T3/fT4 presented stable associations with the level of fasting glucose, glucose at $1 \mathrm{~h} / 2 \mathrm{~h}$ during pregnancy. T3 and T3/fT4 in both the first and second trimesters were positively associated with the risk of GDM, with the larger magnitude of association observed in the second trimester (odds ratio $(\mathrm{OR})=2.50,95 \% \mathrm{Cl}=1.95,3.21$ for T3; OR $=1.09,95 \% \mathrm{Cl}=1.07,1.12$ for T3/fT4). T3 ((AUC) $=0.726,95 \% \mathrm{Cl}=0.698,0.754)$ and T3/fT4 (AUC $=0.724,95 \% \mathrm{Cl}=0.696,0.753$ ) in the first trimester could improve the performance of the prediction model; however, the overall performance is not good. Conclusion: Significant and stable associations of T3, T3/fT4 and glucose metabolism indicators were documented. Both T3 and T3/fT4 improve the performance of the GDM predictive model.
\end{abstract}

Key Words
- longitudinal studies
- triiodothyronine
triiodothyronine/free
thyroxine
- glucose
- pregnancy

\section{Introduction}

The thyroid gland experiences great stress during pregnancy, increasing about $10-40 \%$ in size, with the production of thyroxine (T4) and triiodothyronine (T3) surging by $50 \%$ (1). As a result, thyroid dysfunction such as hypothyroidism, hyperthyroidism and autoantibody positivity complicates up to $17 \%$ of all pregnancies (1). It is well accepted that thyroid function abnormalities have a deleterious impact on pregnancy $(2,3)$. https://ec.bioscientifica.com

https://doi.org/10.1530/EC-21-0088

(c) 2021 The authors Published by Bioscientifica Ltd

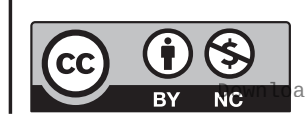

This work is licensed under a Creative Commons Attribution-NonCommercial 4.0 International License. ded from Bioscientifica.com at 04/26/2023 12:49:32PM 
Hyperglycemia during pregnancy typically diagnosed as gestational diabetes mellitus (GDM) is a highly prevalent complication during pregnancy and affects around 15-20\% of pregnancies (4) and the world has been witnessing a rapid increase in GDM incidence (5). Its negative health consequences for women and their offspring occur both during pregnancy (5) and beyond (6).

Thyroid hormones are involved in glucose metabolism and homeostasis through multiple ways $(7,8,9,10)$, and the associations between thyroid dysfunction and glucose abnormality have been well established. However, previous studies mainly focused on thyroid-stimulating hormone (TSH), fT4 and T4. T3 has long been overlooked, even though T3 plays a significant role in glucose regulation (11). In recent years, T3 has gained popularity in the scientific literature due to it being the active metabolic form of thyroid hormone that can induce endogenous glycemic activity (12). Most peripheral T3 is produced in part by the conversion of T4 by two deiodinase enzymes, type 1 and type 2 iodothyronine deiodinases (D1 and D2, respectively) (13), with the rest produced directly by the thyroid gland. Therefore, the ratio of T3 (or fT3) to T4 (or fT4) is attracting attention as well but requires extensive further exploration.

As such, based on repeated measurements from a prospective cohort study, we aimed to: (i) examine the associations of T3 and T3/fT4 with glucose metabolism indicators during pregnancy; (ii) evaluate the performance of the two markers, in the first trimester, as predictors of the risk for GDM.

\section{Material and methods}

\section{Study population}

The Ma'anshan birth cohort study (MABC) is a populationbased prospective study conducted in Ma'anshan city of Anhui Province in China. Between May 2013 and September 2014 , the MABC recruited 3474 pregnant women who were attending their first prenatal health visit. The participants were then followed until labor for a maximum of four visits (i.e. the first, second, and third trimesters and delivery). Of these participants, we first excluded those who lacked information on GDM status and were diagnosed with diabetes before conception, yielding a sample of 3308 women. In addition, we excluded participants without thyroid function data in any trimesters, yielding a final sample of 2723 women. The present study was approved by the ethics committee of Anhui Medical University. Written informed consent was obtained from all pregnant women.

\section{GDM diagnosis}

At 24-28 weeks of gestation, women underwent a GDM screening that consisted of a 'one-step' standardized $75 \mathrm{~g}$ oral glucose tolerance test (OGTT). Venous blood samples were collected at 0,1 , and $2 \mathrm{~h}$ after a glucose load. A positive GDM diagnosis was made when any of the following criteria were met: fasting plasma glucose $\geq 5.1 \mathrm{mmol} / \mathrm{L}$, plasma glucose at $1 \mathrm{~h} \geq 10 \mathrm{mmol} / \mathrm{L}$, or plasma glucose at $2 \mathrm{~h} \geq 8.5 \mathrm{mmol} / \mathrm{L}$ (14). These data, along with fasting glucose in each trimester, were extracted from medical records, and plasma glucose was measured using the glucose oxidase method.

\section{Thyroid hormone measurement}

Blood samples were collected at the first, second, and third health visits. Fasting blood was drawn in the morning before 10:00 $\mathrm{h}$, and serum specimens were isolated and stored at $-80^{\circ} \mathrm{C}$ for further examination. Serum samples were assayed for levels of fT4, T3, thyrotropin (TSH), antithyroperoxidase autoantibody (TPOAb) and antithyroglobulin autoantibody (TgAb) with electrochemiluminescence using the Cobas e411 automated immunoassay platform (Roche Diagnostics $\mathrm{GmbH})$. All samples were tested blindly and each batch included two quality control samples. The coefficients of variation for these thyroid profile assays were all below $10 \%$. The detection limits of fT4, T3, TSH, TPOAb and $\mathrm{TgAb}$ were $0.300 \mathrm{pmol} / \mathrm{L}, 0.300 \mathrm{nmol} / \mathrm{L}, 0.005 \mu \mathrm{IU} / \mathrm{mL}$, $5.0 \mathrm{IU} / \mathrm{mL}$ and $10.0 \mathrm{IU} / \mathrm{mL}$, respectively. TPOAb and $\mathrm{TgAb}$ were considered positive if concentrations were greater than 34.0 and $115.0 \mathrm{IU} / \mathrm{mL}$, respectively.

\section{Covariates}

Extensive data were collected using a structured self-report questionnairethat wasadministered by trained interviewers at each health visit. Smoking in early pregnancy was defined as either ongoing smoking or former smoking that ceased after the woman became aware of her pregnancy status. Alcohol consumption was categorized as never, occasional, or regular. Education level was categorized into five groups: (i) primary school or below; (ii) middle school; (iii) high school; (iv) junior college and (v) undergraduate or above. Monthly income levels (Chinese Yuan) were divided into four groups: (i) < 1000; (ii) 1000-2500; (iii) 2500-4000 and (iv) $\geq 4000$. Seasons for the Northern Hemisphere were defined as spring (i.e. February to April),

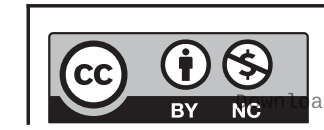


summer (i.e. May to July), fall (i.e. August to October) and winter (i.e. November to January).

\section{Statistical analysis}

After $\log$ transformation, values outside \pm 4 S.D. of the mean were regarded as either laboratory error or undiagnosed disease and were treated as outliers. The outliers were omitted as following, in each trimester: 8 outliers for T3, 17 for fT 4 and 12 for TSH in the first trimester; 1 for T3, 7 for fT 4 and 13 for TSH in the second trimester; 5 for T3, 5 for fT4 and 10 for TSH in the third trimester.

The Mann-Whitney $U$-test was used to compare median concentrations of the different thyroid markers between the GDM and non-GDM groups. Fasting glucose differences between different trimesters were compared using paired samples $t$-test. A generalized estimating equation (GEE) was used to examine the associations of demographic or lifestyle factors with thyroid markers. The multiple informant model with a nonstandard version of GEE was used to examine trimester-specific associations of thyroid markers with fasting plasma glucose, plasma glucose at $1 \mathrm{~h} / 2 \mathrm{~h}$, and the risk of GDM. For fasting plasma glucose, data from three trimesters were analyzed, while for glucose at $1 \mathrm{~h} / 2 \mathrm{~h}$ and the risk of GDM, only data from the first and second trimesters were included in the model.

Stratified analyses of the associations between thyroid markers and the risk of GDM were conducted according to pre-pregnancy BMI, parity, smoking status, TPOAb and $\mathrm{TgAb}$ status using logistic regression. The multiplicative interaction term was evaluated by a likelihood ratio test. Sensitivity analyses of the association between T3, fT4 and T3/fT4 and the risk of GDM were conducted according to the item with which the diagnosis of GDM was made (i.e. high fasting glucose vs high postprandial glucose vs both).

To further specify the clinical significance of T3 and T3/fT4, we built multivariate predictive models along with routine variables (i.e. maternal age, pre-pregnancy BMI, history of family diabetes, gestational seasons, fasting plasma glucose) and compared each of their performances in predicting GDM. Sensitivity, negative predictive value (NPV) and positive predictive value (PPV) were calculated under a fixed specificity of $90.0 \%$ and a fixed false-positive rate of $10 \%$ for different models. The receiver operating characteristic curve (ROC)-area under curve (AUC) was drawn, and the $\mathrm{Z}$ statistic was used to compare the discrimination of different models. Hosmer-Lemeshow goodness of fit test was conducted to assess the calibration of predictive models.
Analyses were conducted using Stata 13.0, R 3.5.1. and MedCalc 18.11.3. Statistical significance was set at $P<0.05$. All tests were two-sided.

\section{Results}

Table 1 presents the characteristics of the 2723 participants in our study. A total of 336 (12.3\%) patients with GDM were documented. There was detectable difference of GDM incidence between individuals with $(n=336 ; 12.3 \%)$ and without thyroid function data $(n=585 ; 15.9 \%)\left(P_{\text {for } x}{ }^{2}=0.02\right)$. The average age was 26.7 years. Fasting glucose level decreases with increasing gestational age ( $P_{\text {for the difference between 1st and 2nd trimester }}<0.001$; $P_{\text {for the difference between 2nd and 3rdtrimester }}<0.001$ ).

Table 2 presents the concentrations of different thyroid hormones in different gestational age groups as a whole and according to GDM status. T3 concentration increases gradually with increasing gestational age, from $2.35 \mathrm{nmol} / \mathrm{L}$ in the first trimester to $2.69 \mathrm{nmol} / \mathrm{L}$ in the third trimester. fT4 drops sharply from the first to the second trimester from 16.7 to $11.9 \mathrm{pmol} / \mathrm{L}$ and increases slightly afterward to $13.3 \mathrm{pmol} / \mathrm{L}$. T3/fT4 increases substantially from 139 in the first trimester to 214 in the second trimester, while decreases a little bit to 202 in the third trimester. TSH increases significantly from $1.58 \mu \mathrm{IU} / \mathrm{mL}$ in the first trimester to $2.42 \mu \mathrm{IU} / \mathrm{mL}$ in the second trimester, while decreases slightly to $2.21 \mu \mathrm{IU} / \mathrm{mL}$ in the third trimester. There are differences in T3 concentrations between the GDM and non-GDM groups across all three periods, as was similar for T3/fT4 concentrations. For fT4 and TSH, the difference was only detected in the second trimester.

Figure 1 shows the consistent percentage of individuals whose concentrations of T3, fT4 and T3/fT 4 were above $75 \%$ or below $25 \%$ across the three trimesters. For T3, the top of $25 \%$ individuals in the first trimester became $11.9 \%$ in the second trimester and $6.3 \%$ in the third trimester. Likewise, the change for fT 4 progressed from $25 \%$ in the first trimester to $12.2 \%$ in the second trimester, and $7 \%$ in the third trimester. T3/fT4 changed from $25 \%$ in the first trimester to $12.3 \%$ in the second trimester and $10.5 \%$ in the third trimester.

Supplementary Table 1 (see section on supplementary materials given at the end of this article) shows the associations between different thyroid markers and related factors. Independent of the adjusted factors, all three thyroid markers were significantly associated with 
Table 1 Characteristics of subjects included in the current study.

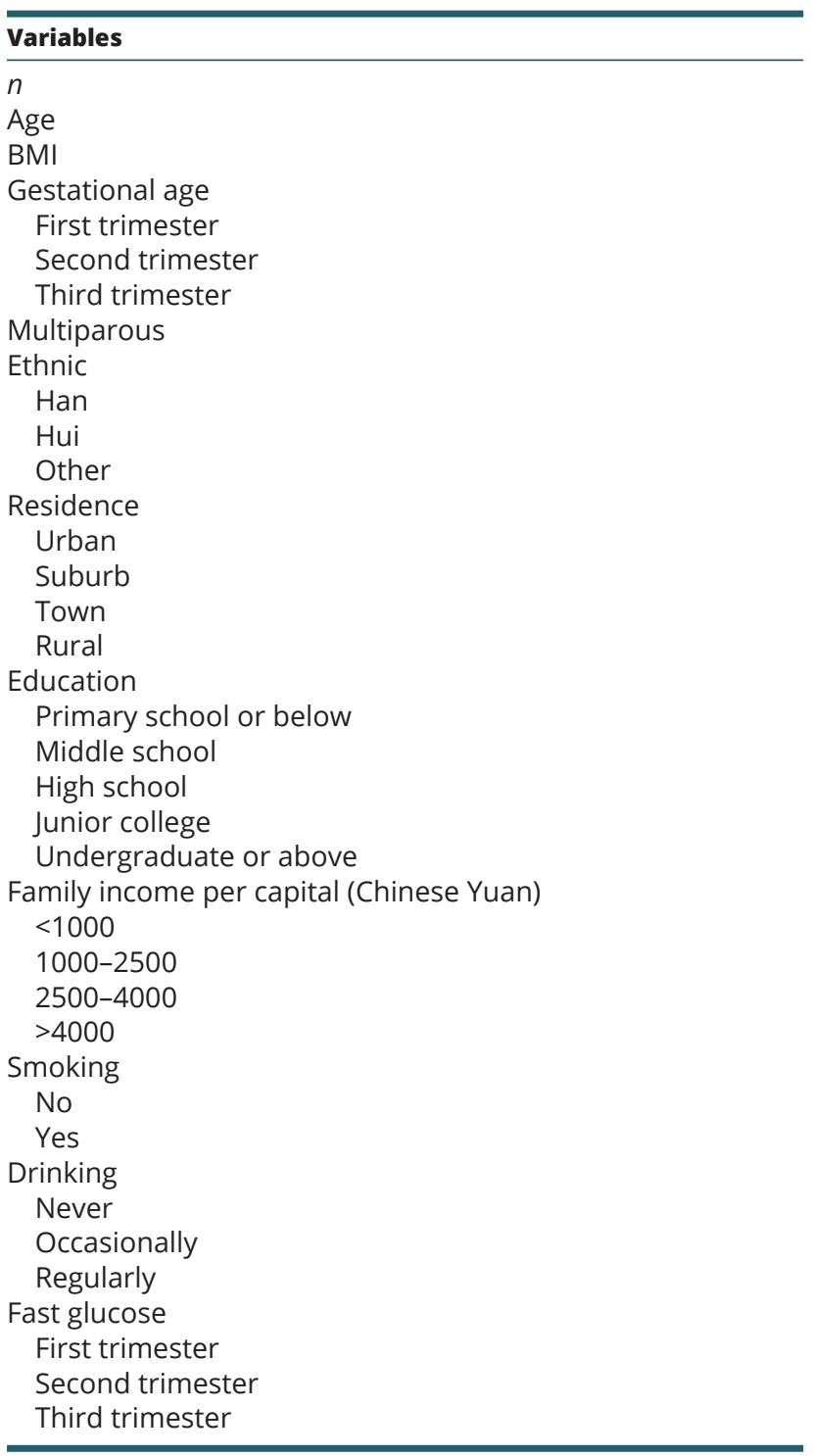

Data are presented as mean (s.D.), or $n$ (\%), as applicable.

pre-pregnancy BMI, gestational weeks, smoking, and sampling seasonality. As for other factors, age was significantly related to T3 and fT 4 concentrations; drinking was significantly associated with $\mathrm{T} 3$ and $\mathrm{T} 3 / \mathrm{fT} 4$ ratio; parity was significantly associated with fT4 concentrations; gestational age and smoking were significantly associated with TSH concentrations.

Table 3 presents trimester-specific associations between thyroid markers and glucose metabolism indicators by GEE. The effect sizes for T3, fT4, T3/fT4 and $\mathrm{TSH}$ are indicated in per $\mathrm{nmol} / \mathrm{L}$, per $\mathrm{pmol} / \mathrm{L}$, per 10 unit and per $\mu \mathrm{IU} / \mathrm{mL}$, respectively. Regarding fasting glucose and glucose at $1 \mathrm{~h} / 2 \mathrm{~h}, \mathrm{~T} 3$ in all periods were significantly associated with all three indicators, except fasting glucose

\begin{tabular}{c}
\hline All subject \\
\hline 2723 \\
$26.7(3.6)$ \\
$20.8(2.8)$ \\
\\
$10.0(2.1)$ \\
$25.6(1.0)$ \\
$34.0(1.0)$ \\
$290(10.7)$ \\
$2682(98.5)$ \\
$23(0.8)$ \\
$18(0.7)$ \\
$2134(78.4)$ \\
$316(11.6)$ \\
$48(1.7)$ \\
$226(8.3)$ \\
$33(1.2)$ \\
$500(18.4)$ \\
$606(22.3)$ \\
$851(31.3)$ \\
$733(26.9)$ \\
$50(1.8)$ \\
$669(24.6)$ \\
$1167(42.9)$ \\
$837(30.7)$ \\
$2614(96.0)$ \\
$109(4.0)$ \\
$2505(92.0)$ \\
$214(7.9)$ \\
$4(0.1)$ \\
$4.7(0.4)$ \\
$4.3(0.5)$ \\
$4.2(0.6)$ \\
\end{tabular}

\begin{tabular}{c}
\hline GDM \\
\hline $336(12.3)$ \\
$28.0(4.3)$ \\
$22.2(3.1)$ \\
\\
$9.9(2.0)$ \\
$25.6(0.9)$ \\
$34.0(1.1)$ \\
$47(14.0)$ \\
$329(97.9)$ \\
$4(1.2)$ \\
$3(0.9)$ \\
$272(81.0)$ \\
$42(12.5)$ \\
$7(2.1)$ \\
$15(4.5)$ \\
$4(1.2)$ \\
$76(22.6)$ \\
$69(20.5)$ \\
$101(30.1)$ \\
$86(25.6)$ \\
$8(2.4)$ \\
$104(31.0)$ \\
$142(42.3)$ \\
$82(24.4)$ \\
$323(96.1)$ \\
$13(3.9)$ \\
$309(92.0)$ \\
$26(7.7)$ \\
$1(0.3)$ \\
$5.0(0.7)$ \\
$4.7(0.9)$ \\
\end{tabular}

\begin{tabular}{c}
\hline Non-GDM \\
\hline $2387(87.7)$ \\
$26.5(3.4)$ \\
$20.6(2.7)$ \\
$10.0(2.1)$ \\
$25.6(1.0)$ \\
$34.0(1.0)$ \\
$243(10.2)$
\end{tabular}

$2353(98.6)$

$19(0.8)$

$15(0.6)$

$1862(78.0)$

$274(11.5)$

$41(1.7)$

$210(8.8)$

$29(1.2)$

$425(17.8)$

$537(22.5)$

$749(31.4)$

$647(27.1)$

$42(1.8)$

$566(23.7)$

$1042(43.7)$

$755(31.6)$

$2291(96.0)$

$96(4.0)$

$2196(92.0)$

$188(7.9)$

$3(0.1)$

$4.7(0.5)$

$4.2(0.5)$

$4.2(0.6)$ in the first trimester, with the largest magnitude of association observed in the second trimester $(\beta=0.16$, $95 \% \mathrm{CI}=0.11,0.20$ for fasting glucose $\beta=0.41,95 \% \mathrm{CI}=$ $0.28,0.55$ for glucose at $1 \mathrm{~h} ; \beta=0.21,95 \% \mathrm{CI}=0.11,0.31$ for glucose at $2 \mathrm{~h}$ ). fT4 in all periods were significantly associated with all the three indicators, except fasting glucose in the third trimester, with the largest magnitude of association observed in the second trimester $(\beta=-0.02$, $95 \% \mathrm{CI}=-0.03,-0.01$ for fasting glucose; $\beta=-0.14$, $95 \% \mathrm{CI}=-0.18,-0.10$ for glucose at $1 \mathrm{~h} ; \beta=-0.08$, $95 \% \mathrm{CI}=-0.11,-0.05$ for glucose at $2 \mathrm{~h}$ ). T3/fT4 in all periods were significantly associated with all the three indicators, with the largest magnitude of association observed in the second trimester $(\beta=-0.02$,

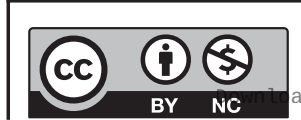


Table 2 Concentrations of thyroid markers according to GDM status in three trimesters.

\begin{tabular}{l}
\hline Variables \\
\hline First trimester \\
T3 \\
fT4 \\
T3/fT4 \\
TSH \\
Second trimester \\
T3 \\
fT4 \\
T3/fT4 \\
TSH \\
Third trimester \\
T3 \\
fT4 \\
T3/fT4 \\
TSH
\end{tabular}

\begin{tabular}{c}
\hline \multicolumn{1}{c}{ Combined } \\
\hline $2.35(1.54,3.53)$ \\
$16.7(12.6,23.5)$ \\
$139(86,221)$ \\
$1.58(0.02,5.59)$ \\
\\
$2.56(1.76,3.56)$ \\
$11.9(9.1,15.3)$ \\
$214(142,328)$ \\
$2.42(0.70,5.89)$ \\
$2.69(1.68,3.75)$ \\
$13.3(9.5,17.2)$ \\
$202(128,303)$ \\
$2.21(0.51,5.57)$ \\
\hline
\end{tabular}

\begin{tabular}{c}
\hline Non-GDM \\
\hline $2.33(2.01,2.68)$ \\
$16.7(15.4,18.4)$ \\
$137(117,162)$ \\
$1.58(0.87,2.54)$ \\
\\
$2.53(2.26,2.84)$ \\
$12.0(11.0,13.0)$ \\
$211(184,243)$ \\
$2.46(1.74,3.35)$ \\
$2.68(2.30,3.05)$ \\
$13.3(11.9,14.8)$ \\
$199(172,233)$ \\
$2.21(1.55,3.08)$ \\
\hline
\end{tabular}

\begin{tabular}{c}
\hline GDM \\
\hline $2.50(2.14,2.84)$ \\
$16.7(15.1,18.0)$ \\
$148(125,175)$ \\
$1.58(0.95,2.39)$ \\
$2.78(2.46,3.15)$ \\
$11.5(10.5,12.5)$ \\
$240(209,276)$ \\
$2.13(1.56,3.02)$ \\
$2.82(2.46,3.20)$ \\
$13.2(11.8,14.4)$ \\
$214(185,248)$ \\
$2.19(1.51,2.98)$ \\
\hline
\end{tabular}

\begin{tabular}{c}
\hline $\boldsymbol{P}$ \\
\hline \\
$<0.001$ \\
0.09 \\
$<0.001$ \\
0.87 \\
\\
$<0.001$ \\
$<0.001$ \\
$<0.001$ \\
$<0.001$ \\
$<0.001$ \\
$<0.24$ \\
$<0.001$ \\
0.21
\end{tabular}

Combined samples are presented as median (2.5th and 97.5th percentile). GDM and non-GDM groups are presented as median (25th and 75th percentile). Unit of T3, fT4 and TSH was $\mathrm{nmol} / \mathrm{L}$, pmol/L and $\mu \mathrm{lU} / \mathrm{mL}$. Boldface indicates statistically significant.

$95 \% \mathrm{CI}=-0.03,-0.01$ for fasting glucose; $\beta=-0.14$, $95 \% \mathrm{CI}=-0.18,-0.10$ for glucose at $1 \mathrm{~h} ; \beta=-0.08$, $95 \% \mathrm{CI}=-0.11,-0.05$ for glucose at $2 \mathrm{~h}$ ); TSH in both first and second trimester was significantly associated with glucose at $1 / 2 \mathrm{~h}$. In the first trimester, only $\mathrm{T} 3$ $(\mathrm{OR}=1.67,95 \% \mathrm{CI}=1.33,2.10)$ and $\mathrm{T} 3 / \mathrm{fT} 4(\mathrm{OR}=1.08$, $95 \% \mathrm{CI}=1.04,1.12$ ) were significantly associated with the risk of GDM, while in the second trimester, $\mathrm{T} 3(\mathrm{OR}=2.50$, $95 \% \mathrm{CI}=1.95,3.21)$, fT $4 \quad(\mathrm{OR}=0.89,95 \% \mathrm{CI}=0.82$, $0.96)$, T3/fT4 $(\mathrm{OR}=1.09,95 \% \mathrm{CI}=1.07,1.12)$ and $\mathrm{TSH}$ $(\mathrm{OR}=0.91,95 \% \mathrm{CI}=0.83,0.99)$ in the second trimester were all significantly associated with the risk of GDM.

Stratified analyses of associations between three thyroid markers and the risk of GDM are presented in Supplementary Table 2. Sensitivity analyses of the associations of three thyroid markers and the risk of GDM according to the item with which the GDM is diagnosed are shown in Supplementary Table 3.

Because T3 and T3/fT4 in the first trimester were both significantly associated with the risk of GDM, we further assessed their performances in predicting GDM. As shown in Table 4 and Fig. 2, adding T3 and T3/fT4 in the models respectively, the performance of model 3 (AUC $=0.726$, $95 \% \mathrm{CI}=0.698,0.754)$ and model 4 (AUC $=0.724$, $95 \% \mathrm{CI}=0.696,0.753)$ improved and showed comparable medium degree of discriminative ability, and the AUC of the models ranked as model $3(0.726)>$ model 4 $(0.724)>$ model $2(0.710)>$ model $1(0.703)$. As shown in Supplementary Table 4, the comparison between model 3 and model $1(P=0.004)$; model 3 and model $2(P=0.02)$; model 4 and model $1(P=0.01)$; model 4 and model $2(P=0.04)$ reached statistical significance.
The Hosmer-Lemeshow goodness of fit test indicated that the calibration capability of the four models was good $(P$ value $>0.05)$. However, the calibration diagram indicated that the calibration capability of model 4 (Fig. 3B) was better than model 3 (Fig. 3A).The best multivariate predictive model of GDM including T3 (or T3/fT4), maternal age, pre-pregnancy BMI, fasting plasma glucose in the first trimester, sampling gestational week, the season of conception and family history of diabetes.

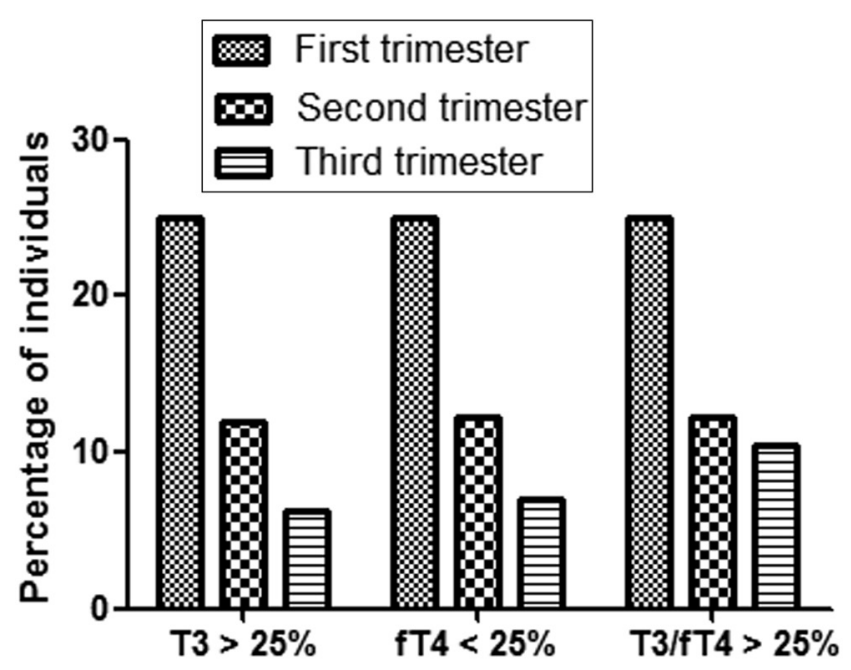

Figure 1

Consistency of the three thyroid markers across three trimesters of pregnancy. The first bar indicates the highest or lowest $25 \%$. The following two bars indicate the percentage of individuals who are above $75 \%$ or below $25 \%$ in the first trimester, persisting through the second and third trimesters. 


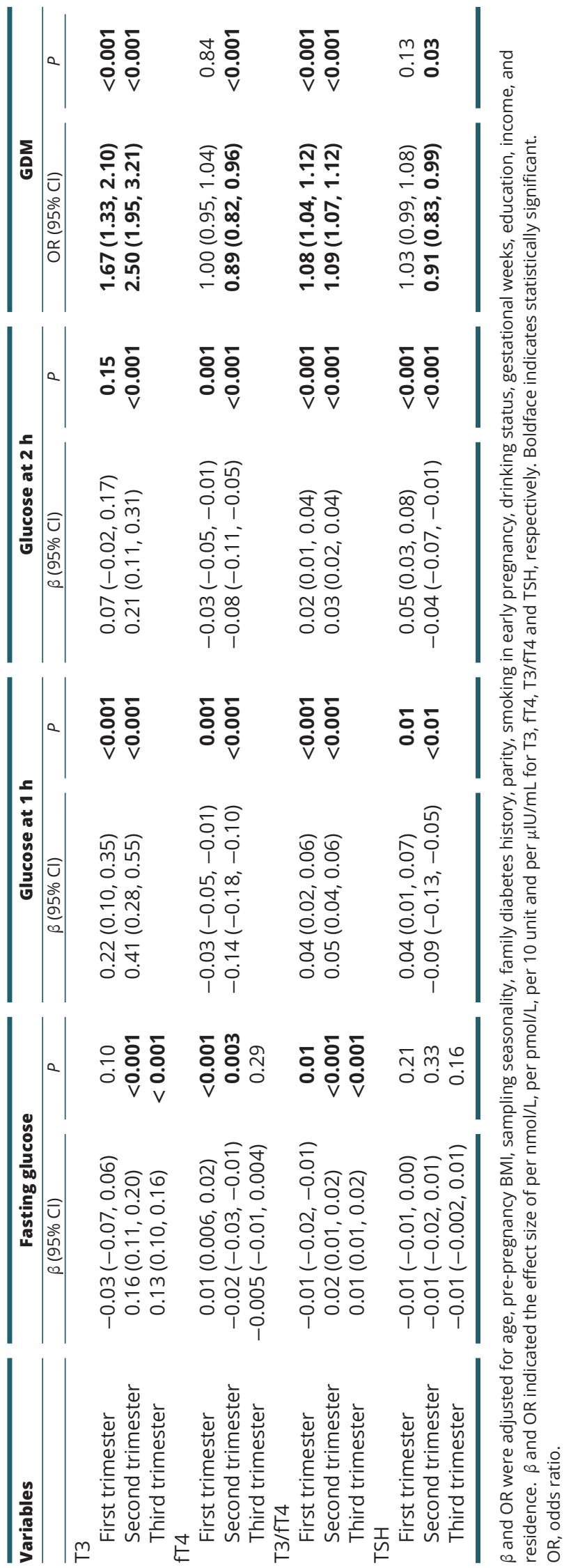

https://ec.bioscientifica.com https://doi.org/10.1530/EC-21-0088

(c) 2021 The authors Published by Bioscientifica Ltd

\section{Discussion}

Our current study demonstrated trimester-specific associations between T3, fT4 and T3/fT4 with glucose metabolism indicators, with the largest magnitude of association observed in the second trimester for all three thyroid markers, and the most stable associations for T3/ fT4 during pregnancy. Along with routine factors, T3 and T3/fT4 in the first trimester could improve the performance of the GDM prediction model and exhibited a comparable degree of discrimination ability and good calibration.

Our study echoed, and built upon, a recent nested casecontrol study from the US which indicated that both fT3 and fT3/fT4 were significantly positively associated with the risk of GDM (15). T3 is the primary active hormone involved in glucose metabolism (16) (12), yet it has long been neglected regarding its association with GDM. Given low fT4 hormonal activity, it is unlikely to influence plasma glucose directly. As a result, two studies $(17,18)$ reporting the associations between $\mathrm{fT} 4$ concentrations and the risk of GDM proposed that deiodinase activity might be the underlying factor. Only a handful of longitudinal studies $(15,19,20)$ explored the associations of T3, fT3, or deiodinase activity (often indicated as fT3/fT4 or T3/fT4) with the risk of GDM. Neither a study from the United Arab Emirates with 301 participants (19) nor a study from Greece with 1170 participants (20) indicated that early pregnancy fT3 concentrations were associated with the risk of GDM. To our knowledge, the remaining studies were all cross-sectional in nature. Other than dichotomous GDM status $(21,22)$, some also focused on the glucose metabolic profile $(23,24,25)$, and all reported significant associations between fT3/fT4 or T3/fT4 and glucose metabolism-related indicators.

In addition to stimulating endogenous glucose production (12), T3 could be involved in glucose metabolism in various ways. For example, T3 can positively regulate mitochondrial function and induce mitochondria biogenesis $(26,27)$, dysfunction of the latter emerged as a new insight into the etiology of diabetes and glucose abnormality (11). In addition, T3 can exert an important effect on the control of energy expenditure. For instance, under the effect of T3, skeletal muscle energy metabolism is switched into oxidative mitochondrial metabolism mediated by PGC-1 $\alpha$ (28), which plays an indispensable role in glucose and fatty acid metabolism (29). Furthermore, T3 can increase glucose uptake independent of insulin in skeletal muscle cells, such as through rapidly increasing GLUT4 expression (30), and the latter plays an important role in whole-body glucose homeostasis. 
Table 4 Performance of multivariate predictive model of GDM including thyroid hormones and routine variables at a fixed false-positive rate of $10 \%$.

\begin{tabular}{|c|c|c|}
\hline Models & AUC $(95 \% \mathrm{Cl})$ & Sensitivity (\%) \\
\hline Model 1 & $0.703(0.686,0.720)$ & 26.5 \\
\hline Model 2 & $0.710(0.693,0.729)$ & 29.2 \\
\hline Model 3 & $0.726(0.709,0.743)$ & 30.8 \\
\hline Model 4 & $0.724(0.707,0.741)$ & 32.4 \\
\hline
\end{tabular}

\begin{tabular}{c}
\hline Specificity $(\%)$ \\
\hline 90.0 \\
90.0 \\
90.0 \\
90.0
\end{tabular}

\begin{tabular}{c}
\hline NPV $(\%)$ \\
\hline 89.7 \\
90.0 \\
90.2 \\
90.4 \\
\hline
\end{tabular}

\begin{tabular}{c}
\hline PPV $(\%)$ \\
\hline 27.1 \\
29.1 \\
30.1 \\
31.3 \\
\hline
\end{tabular}

\begin{tabular}{cccc}
\cline { 1 - 1 } Cut-off & & $\boldsymbol{P}$ \\
\hline 0.208 & & 0.060 \\
0.211 & & 0.477 \\
0.215 & & 0.072 \\
0.215 & & 0.685 \\
\hline
\end{tabular}

$P$ value of Hosmer-Lemeshow good of fit test. Model 1 including maternal age, pre-pregnancy BMI, history of family diabetes, and season of conception; Model 2 based on model 1, plus fasting plasma glucose and sampling gestational week; Model 3 based on model 2, plus T3; Model 4 based on model 2, plus T3/fT4.

AUC, area under curve; NPV, negative predictive value; PPV, positive predictive value.

Collectively, our data strongly support the authentic roles of T3 in the etiology of GDM.

T3 and T3/fT4 in the first trimester could improve the performance of the GDM prediction model; however, even along with routine factors, judging from the NPV and PPV of the predictive model, the performance of the model is not good. A recent review (31) indicated that universal vs risk-based screening for thyroid problems did not affect the risk of GDM, and the evidence was originally derived from a trial (32) that included 4516 women. All women from the trial in the universal screening group and high-risk women in the case finding group had their sera immediately tested for TSH, fT4, and TPOAb

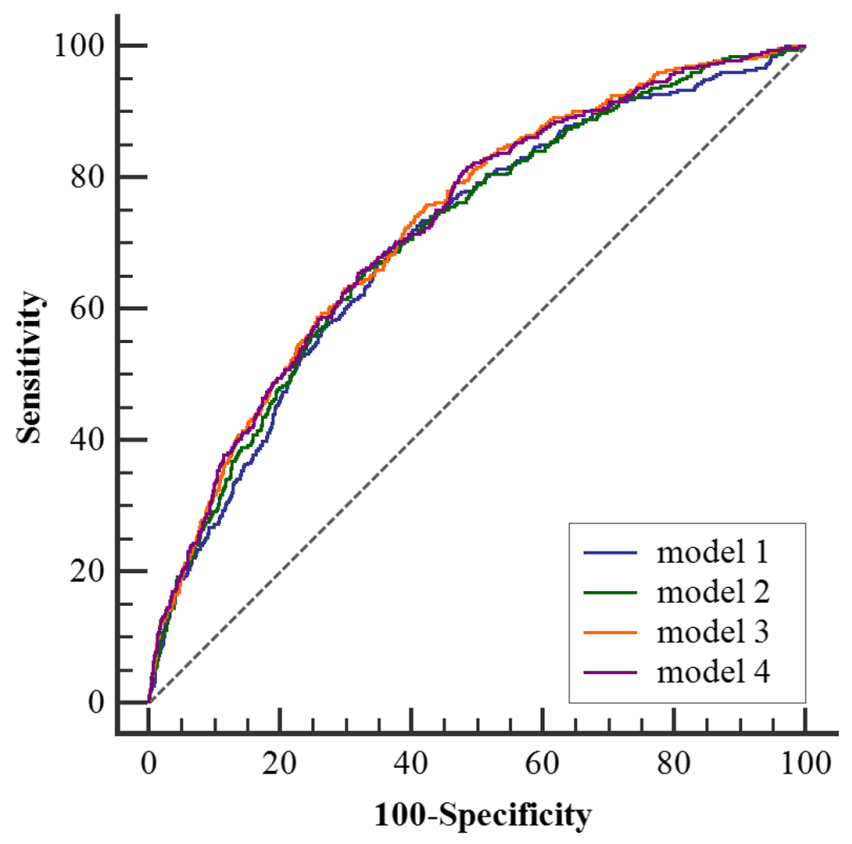

Figure 2

Comparison of multivariate predictive models. Model 1 including maternal age, pre-pregnancy BMI, history of family diabetes, and season of conception; Model 2 based on model 1, plus fasting plasma glucose and sampling gestational week; Model 3 based on model 2, plus T3; Model 4 based on model 2, plus T3/fT4. to identify thyroid dysfunction; however, subsequent medication interventions did not benefit those women in preventing GDM. Our study does not conflict with this trial, because we emphasized the importance of $\mathrm{T} 3$ and T3/fT4, although the extent to which the screening of
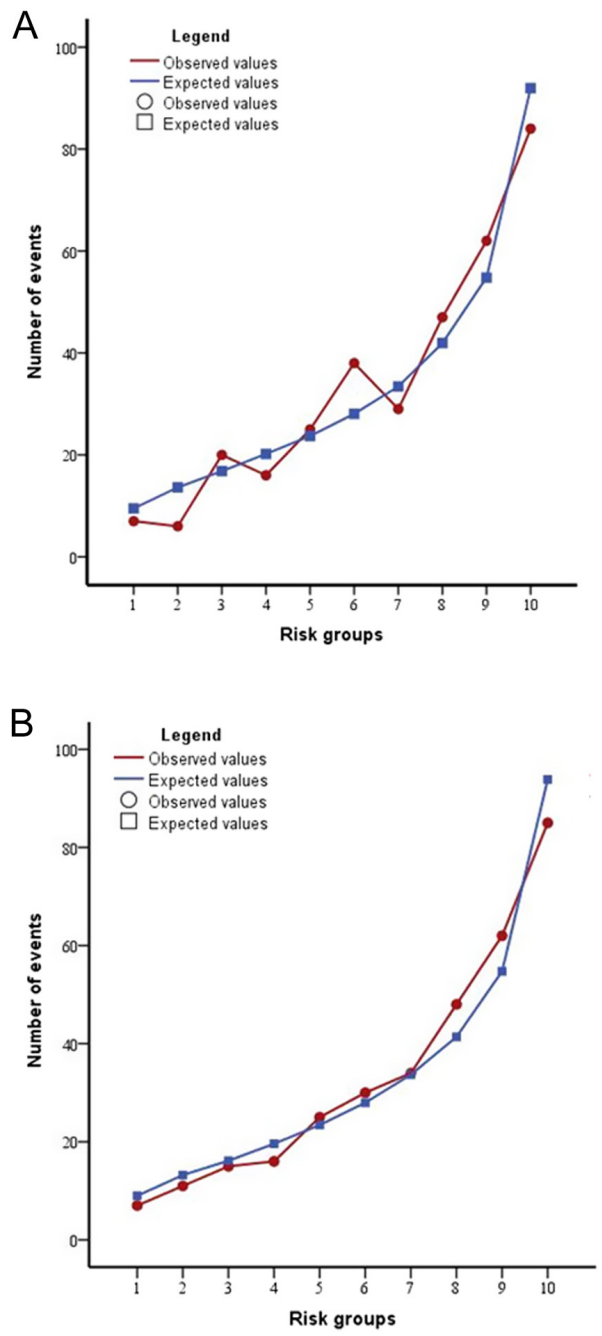

Figure 3

Calibration chart of multivariate predictive models including thyroid hormones. (A) Model including T3; (B) Model including T3/fT4.

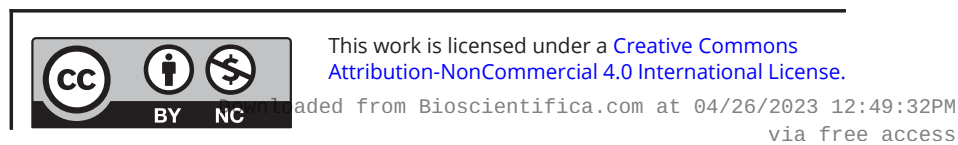


$\mathrm{T} 3$ and T3/fT4 in the first trimester could benefit women, regarding GDM prevention, is still uncertain and requires the collection of more data. Although OGTT becomes a routine test, given its inconvenience, not every woman may opt to undergo this test. While thyroid function TSH and T4 testing is being routinely adopted in early pregnancy around China, our study justifies adding T3 into this routine, which would help identify high-risk individuals for GDM. Recently, a large sample-sized study (33) from China attempted to build a GDM prediction model. The study team selected 17 variables from 73 extracted variables, of which thyroid function (i.e. TT3 and TT4) was included. Interestingly, the results indicated the TT3 and TT4 levels had better predictive power than FT3 and FT4.

Another interesting finding was that the consistency of fasting glucose throughout pregnancy is better than thyroid markers. Previous studies on thyroid function mainly focused on early pregnancy and lacked longitudinal data to illustrate the natural course of gestational thyroid function. Our study showed that individuals with T3, fT4, and T3/fT4 levels among the highest or lowest $25 \%$ of the populations, in the first trimester, persisted in $6.3,7.0$, and $10.5 \%$ of the population in the third trimester is in line with a recent study (34) which indicated that early pregnancy thyroid disease only persists until the third trimester in $8.4-24.8 \%$ of cases when left untreated. So, the necessity of further thyroid function tests during pregnancy should not only be decided by previous measures.

Our study has several strengths. GDM diagnoses are often made during the second trimester (24-28 gestational weeks), which presents a limited time for an intervention. Our study innovatively proposed including thyroid function in the GDM prediction model, which could help identify high-risk individuals at an early stage. In addition, taking advantage of the MABC longitudinal data with repeated measurements, we were able to assess the persistence of the thyroid markers across three trimesters, which could aid clinicians in making decisions regarding thyroid function testing during pregnancy. Some limitations also need to be noted. First, iodine status at the individual level was not determined in our study, which could have influenced our results to some extent. However, according to 2019 statistics from the Centers for Disease Control and Prevention (CDC), people in Ma'anshan city are generally exposed to appropriate iodine conditions, and the median urinary iodine concentration of women is $174.05 \mu \mathrm{g} / \mathrm{L}$. Secondly, the level of fT3 was not evaluated in our study, which limits comparisons of its importance with that of T3. Thirdly, since we do not have data on thyroxine- binding globulin (TGB), this could affect the interpretation of our results.

Our current study documented significant and stable associations between T3, T3/fT4 and glucose metabolism indicators and both T3 and T3/fT4 could improve the performance of the GDM prediction model.

\section{Supplementary materials}

This is linked to the online version of the paper at https://doi.org/10.1530/ EC-21-0088.

\section{Declaration of interest}

The authors declare that there is no conflict of interest that could be perceived as prejudicing the impartiality of the research reported.

\section{Funding}

This work is supported by two grants from National Natural Science Foundation of China (NSCF-81602866; NSCF-81573168). The funder was not involved in study design, data collection, data analysis, and manuscript preparation and/or publication decisions.

\section{Author contribution statement}

Beibei Zhu: formal analysis and writing original draft; Yan Han: resources; Fen Deng: methodology; Shuangqin Yan and Kun Huang: project administration; Jiahu Hao and Peng Zhu: writing review and editing; Fangbiao Tao and Beibei Zhu: funding acquisition. Fangbiao Tao: conceptualization and supervision.

\section{References}

1 Alexander EK, Pearce EN, Brent GA, Brown RS, Chen H, Dosiou C, Grobman WA, Laurberg P, Lazarus JH, Mandel SJ, et al. 2017 Guidelines of the American Thyroid Association for the diagnosis and management of thyroid disease during pregnancy and the postpartum. Thyroid 201727 315-389. (https://doi.org/10.1089/thy.2016.0457)

2 Consortium on Thyroid and Pregnancy-Study Group on Preterm Birth, Korevaar TIM, Derakhshan A, Taylor PN, Meima M, Chen L, Bliddal S, Carty DM, Meems M, Vaidya B, et al. Association of thyroid function test abnormalities and thyroid autoimmunity with preterm birth: a systematic review and meta-analysis. JAMA 2019322 632-641. (https://doi.org/10.1001/jama.2019.10931)

3 Derakhshan A, Peeters RP, Taylor PN, Bliddal S, Carty DM, Meems M, Vaidya B, Chen L, Knight BA, Ghafoor F, et al. Association of maternal thyroid function with birthweight: a systematic review and individualparticipant data meta-analysis. Lancet: Diabetes and Endocrinology 2020 8 501-510. (https://doi.org/10.1016/S2213-8587(20)30061-9)

4 American Diabetes Association, Berg EG, Saraco M, Petersen MP, Uelmen S, Robinson S \& Amer Diabet A. 2. Classification and diagnosis of diabetes: standards of medical care in Diabetes-2019. Diabetes Care 201942 S13-S28. (https://doi.org/10.2337/dc19-S002)

5 Natamba BK, Namara AA \& Nyirenda MJ. Burden, risk factors and maternal and offspring outcomes of gestational diabetes mellitus (GDM) in sub-Saharan Africa (SSA): a systematic review and metaanalysis. BMC Pregnancy and Childbirth 201919 450. (https://doi. org/10.1186/s12884-019-2593-z)

6 Perng W, Ringham BM, Smith HA, Michelotti G, Kechris KM \& Dabelea D. A prospective study of associations between in utero exposure to gestational diabetes mellitus and metabolomic profiles 
during late childhood and adolescence. Diabetologia 202063 296-312. (https://doi.org/10.1007/s00125-019-05036-z)

7 Weinstein SP \& Haber RS. Glucose transport stimulation by thyroid hormone in ARL 15 cells: partial role of increased GLUT1 glucose transporter gene transcription. Thyroid 19933 135-142. (https://doi. org/10.1089/thy.1993.3.135)

8 Cortizo AM, Chazenbalk GD, de Gagliardino EE, García ME, Pisarev MA \& Gagliardino JJ. Thyroid hormone binding and deiodination by pancreatic islets: relationship with the in vitro effect upon insulin secretion. Acta Endocrinologica 1987 116 66-72. (https:// doi.org/10.1530/acta.0.1160066)

9 Wrutniak C, Cassar-Malek I, Marchal S, Rascle A, Heusser S, Keller JM, Flechon J, Dauca M, Samarut J \& Ghysdael J. A 43-kDa protein related to c-Erb A alpha 1 is located in the mitochondrial matrix of rat liver. Journal of Biological Chemistry 1995270 16347-16354. (https://doi. org/10.1074/jbc.270.27.16347)

10 Casas F, Rochard P, Rodier A, Cassar-Malek I, Marchal-Victorion S, Wiesner RJ, Cabello G \& Wrutniak C. A variant form of the nuclear triiodothyronine receptor c-ErbAalpha1 plays a direct role in regulation of mitochondrial RNA synthesis. Molecular and Cellular Biology 199919 7913-7924. (https://doi.org/10.1128/MCB.19.12.7913)

11 Crunkhorn S \& Patti ME. Links between thyroid hormone action, oxidative metabolism, and diabetes risk? Thyroid 200818 227-237. (https://doi.org/10.1089/thy.2007.0249)

12 Betley S, Peak M \& Agius L. Triiodo-L-thyronine stimulates glycogen synthesis in rat hepatocyte cultures. Molecular and Cellular Biochemistry 1993120 151-158. (https://doi.org/10.1007/BF00926088)

13 Gereben B, Zavacki AM, Ribich S, Kim BW, Huang SA, Simonides WS, Zeold A \& Bianco AC. Cellular and molecular basis of deiodinaseregulated thyroid hormone signaling. Endocrine Reviews 200829 898-938. (https://doi.org/10.1210/er.2008-0019)

14 American Diabetes Association. Association AD: standards of medical care in diabetes-2013. Diabetes Care 201336 (Supplement 1) S11-S66. (https://doi.org/10.2337/dc13-S011)

15 Rawal S, Tsai MY, Hinkle SN, Zhu YY, Bao W, Lin Y, Panuganti P, Albert PS, Ma RCW \& Zhang CL. A longitudinal study of thyroid markers across pregnancy and the risk of gestational diabetes. Journal of Clinical Endocrinology and Metabolism 2018103 2447-2456. (https:// doi.org/10.1210/jc.2017-02442).

16 Potenza M, Via MA \& Yanagisawa RT. Excess thyroid hormone and carbohydrate metabolism. Endocrine Practice 200915 254-262. (https://doi.org/10.4158/EP.15.3.254)

17 Haddow JE, Craig WY, Neveux LM, Palomaki GE, LambertMesserlian G, Malone FD \& D'Alton ME. First second trimester R. Free thyroxine during early pregnancy and risk for gestational diabetes. PLOS ONE 20161111

18 Yang S, Shi FT, Leung PCK, Huang HF \& Fan JX. Low thyroid hormone in early pregnancy is associated with an increased risk of gestational diabetes mellitus. Journal of Clinical Endocrinology and Metabolism 2016 101 4237-4243. (https://doi.org/10.1210/jc.2016-1506)

19 Agarwal MM, Dhatt GS, Punnose J, Bishawi B \& Zayed R. Thyroid function abnormalities and antithyroid antibody prevalence in pregnant women at high risk for gestational diabetes mellitus. Gynecological Endocrinology 200622 261-266. (https://doi. org $/ 10.1080 / 09513590600630470)$

20 Karakosta P, Alegakis D, Georgiou V, Roumeliotaki T, Fthenou E, Vassilaki M, Boumpas D, Castanas E, Kogevinas M \& Chatzi L. Thyroid dysfunction and autoantibodies in early pregnancy are associated with increased risk of gestational diabetes and adverse birth outcomes. Journal of Clinical Endocrinology and Metabolism 201297 4464-4472. (https://doi.org/10.1210/jc.2012-2540)

21 Haddow JE, Lambert-Messerlian G, Eklund E, Neveux LM \& Palomaki GE. Peripheral deiodinase activity: a potential explanation for the association between maternal weight and gestational hyperglycemia. Obstetric Medicine 201811 73-78. (https://doi.org/10.1177/1753495X17733223)

22 Haddow JE, Metzger BE, Lambert-Messerlian G, Eklund E, Coustan D, Catalano P \& Palomaki GE. Maternal BMI, peripheral deiodinase activity, and plasma glucose: relationships between white women in the HAPO study. Journal of Clinical Endocrinology and Metabolism 2019 104 2593-2600. (https://doi.org/10.1210/jc.2018-02328)

23 Bassols J, Prats-Puig A, Soriano-Rodriguez P, Garcia-Gonzalez MM, Reid J, Martinez-Pascual M, Mateos-Comeron F, de Zegher F, Ibanez L \& Lopez-Bermejo A. Lower free thyroxin associates with a less favorable metabolic phenotype in healthy pregnant women. Journal of Clinical Endocrinology and Metabolism 201196 3717-3723. (https://doi org/10.1210/jc.2011-1784)

24 Jing S, Xiaoying D, Ying X, Rui L, Mingyu G, Yuting C, Yanhua Y, Yufan W, Haiyan S \& Yongde P. Different levels of thyroid hormones between impaired fasting glucose and impaired glucose tolerance: free T3 affects the prevalence of impaired fasting glucose and impaired glucose tolerance in opposite ways. Clinical Endocrinology 201480 890-898. (https://doi.org/10.1111/cen.12384)

25 Knight BA, Shields BM, Hattersley AT \& Vaidya B. Maternal hypothyroxinaemia in pregnancy is associated with obesity and adverse maternal metabolic parameters. European Journal of Endocrinology 2016 174 51-57. (https://doi.org/10.1530/EJE-15-0866)

26 Coppola A, Liu ZW, Andrews ZB, Paradis E, Roy MC, Friedman JM, Ricquier D, Richard D, Horvath TL, Gao XB, et al. A central thermogenic-like mechanism in feeding regulation: an interplay between arcuate nucleus T3 and UCP2. Cell Metabolism 20075 21-33 (https://doi.org/10.1016/j.cmet.2006.12.002)

27 Tata JR, Ernster L, Lindberg O, Arrhenius E, Pedersen S \& Hedman R. The action of thyroid hormones at the cell level. Biochemical Journal 196386 408-428. (https://doi.org/10.1042/bj0860408)

28 Irrcher I, Adhihetty PJ, Sheehan T, Joseph AM \& Hood DA. PPAR $\gamma$ coactivator-1 $\alpha$ expression during thyroid hormone- and contractile activity-induced mitochondrial adaptations. American Journal of Physiology: Cell Physiology 2003284 C1669-C1677. (https://doi. org/10.1152/ajpcell.00409.2002)

29 Handschin C \& Spiegelman BM. The role of exercise and PGC1 alpha in inflammation and chronic disease. Nature 2008 454 463-469. (https://doi.org/10.1038/nature07206)

30 Brunetto EL, Teixeira Sda S, Giannocco G, Machado UF \& Nunes MT. T-3 rapidly increases SLC2A4 gene expression and GLUT4 trafficking to the plasma membrane in skeletal muscle of rat and improves glucose homeostasis. Thyroid 201222 70-79. (https://doi.org/10.1089/ thy.2010.0409)

31 Griffith RJ, Alsweiler J, Moore AE, Brown S, Middleton P, Shepherd E \& Crowther CA. Interventions to prevent women from developing gestational diabetes mellitus: an overview of Cochrane Reviews. Cochrane Database of Systematic Reviews 20206 CD012394. (https://doi. org/10.1002/14651858.CD012394.pub3).

32 Negro R, Schwartz A, Gismondi R, Tinelli A, Mangieri T \& StagnaroGreen A. Universal screening versus case finding for detection and treatment of thyroid hormonal dysfunction during pregnancy. Journal of Clinical Endocrinology and Metabolism 201095 1699-1707. (https:// doi.org/10.1210/jc.2009-2009)

33 Wu YT, Zhang CJ, Mol BW, Kawai A, Li C, Chen L, Wang Y, Sheng JZ, Fan JX, Shi Y, et al. Early prediction of gestational diabetes mellitus in the Chinese population via advanced machine learning. Journal of Clinical Endocrinology and Metabolism 2021106 e1191-e1205. (https:// doi.org/10.1210/clinem/dgaa899)

34 Fan J, Zhang Y, Zhang C, Barjaktarovic M, Yang X, Peeters RP, Huang HF \& Korevaar TIM. Persistency of thyroid dysfunction from early to late pregnancy. Thyroid 201929 1475-1484. (https://doi. org/10.1089/thy.2019.0115)

Received in final form 16 May 2021

Accepted 2 June 2021

Accepted Manuscript published online 2 June 2021 https://ec.bioscientifica.com https://doi.org/10.1530/EC-21-0088 (c) 2021 The authors Published by Bioscientifica Ltd
This work is licensed under a Creative Commons Attribution-NonCommercial 4.0 International License. ded from Bioscientifica com at 04/26/2023 12:49:32PM 